\title{
Das Kind als Projekt seiner Eltern
}

\section{Katharina Glatz}

Leitende Ärztin Institut für Pathologie, Universitätsspital Basel, Vizepräsidentin Forum Medizin und Philosophie

Bei diesem Text handelt es sich um eine gekürzte und leicht modifizierte Form einer Qualifikationsarbeit, die im Rahmen des CAS Philosophie und Medizin an der Universität Luzern entstanden ist.
* Der Begriff Eugenik kann unterschiedlich verwendet werden. In diesem Text wird er im Sinne von b und c verwendet:

a. Klassische Verwendung: staatliche Fortpflanzungspolitik zur Verbesserung des Genpools der Bevölkerung.

b. Liberale Eugenik: Verbesserung/Optimierung nicht der gesamten Bevölkerung, sondern nur des eigenen Nachwuchses, potentiell ähnliche Effekte wie a.

c. Praktiken mit eugenischen Folgen, potentiell ähnliche Effekte wie $a$. und b.

Korrespondenz:

PD Dr. Katharina Glatz

Universitätsspital Basel

Schönbeinstrasse 40

CH-4031 Basel

Tel. 0612652880

Fax 0612653194

katharina.glatz[at]unibas.ch http://fomep.ch
Rasche Fortschritte in der Methodik der pränatalen Diagnostik und der Reproduktionsmedizin, die bereits jetzt oder in wenigen Jahren für die breite Anwendung zur Verfügung stehen, werden uns noch nie da gewesene Möglichkeiten zur gezielten positiven Selektion von Embryonen mit erwünschten Erbanlagen (positive Eugenik ${ }^{*}$ ) oder zur gezielten Elimination von Embryonen mit unerwünschten Erbanlagen (negative Eugenik) eröffnen. Forschungsanstrengungen zur Perfektionierung pränataler Diagnosemethoden und die daraus folgende eugenische Selektion sind motiviert durch den Wunsch nach einem gesunden Kind, das immer höheren Normvorstellungen genügen soll. Dieser Anspruch setzt sich nach der Geburt fort in der zunehmend geäusserten Vorstellung heutiger Eltern von ihrem Kind als ihrem Projekt und in einer zunehmenden Pathologisierung und Medikalisierung minimal normabweichenden kindlichen Verhaltens. In dieser Arbeit soll untersucht werden, inwiefern die Anwendung moderner Methoden der pränatalen Diagnostik und die Vorstellung vom Kind als Projekt der Eltern im Widerspruch stehen zu der von Immanuel Kant aufgestellten Selbstzweckformel und dem daraus abgeleiteten Instrumentalisierungsverbot.

\section{Was sagen uns die Betroffenen? «Wir sind Frauen mit Turner-Syndrom; besondere Frauen, starke Frauen, die einen ungleichen Kampf gewonnen haben; nur zwei Prozent von uns überleben, um der Welt als Frauen mit Turner-Syndrom ins Gesicht zu sehen; deshalb sind wir verdammt glücklich zu leben!}

\section{Nun, Herr Eugeniker,}

Sie brüten ja gerade die neue Superrasse aus; wagen Sie nicht uns zu erzählen, dass wir fehlerhaft und minderwertig sind und deshalb besser tot wären.» [1]

Dies ist der Auszug aus einem Gedicht einer Frau mit Turner-Syndrom (Monosomie X). Rasche Fortschritte in der Methodik der pränatalen Diagnostik und der Reproduktionsmedizin, die bereits jetzt oder in wenigen Jahren für die breite Anwendung zur Verfügung stehen, werden uns noch nie dagewesene Möglichkeiten zur gezielten positiven Selektion von
Embryonen mit erwünschten Erbanlagen oder zur gezielten Elimination von Embryonen mit unerwünschten Erbanlagen eröffnen. Die Monosomie X ist eine chromosomale Aberration, die sich mit den neuen Methoden der Pränataldiagnostik einfach feststellen lassen wird. Das Gedicht lässt keinen Zweifel an der Tatsache, dass Frauen mit Turner-Syndrom ein erfülltes und selbstbestimmtes Leben führen können und einer negativen Selektion von Embryonen mit Monosomie X keineswegs zustimmen würden.

Die Grenzen zwischen positiver und negativer Eugenik werden zunehmend fliessend, wenn Embryonen beim Nachweis bestimmter potentiell krankheitsverursachender Erbanlagen, deren Schweregrad nicht voraussehbar ist, gezielt abgetrieben werden.

\section{Das Kind als Projekt seiner Eltern}

Der Zeitpunkt einer Schwangerschaft ist seit wenigen Jahrzehnten dank sehr verlässlicher antikonzeptiver Methoden und immer erfolgreicherer Methoden der Reproduktionsmedizin zunehmend planbar geworden. Die Nachwuchsplanung beschränkt sich aber immer mehr nicht nur auf den optimalen Zeitpunkt der Schwangerschaft, sondern beinhaltet zunehmend auch den Wunsch nach gesundem Nachwuchs. Wer ein krankes Kind zur Welt bringt, hat zu wenig gut geplant. In einer individualisierten Gesellschaft, die grossen Wert auf die Autonomie des Individuums legt, wird die Reproduktionsfreiheit der Eltern gegenüber staatlichen Eingriffen und gegenüber den noch nicht absolut gültigen Grundrechten des Embryos vielfach bevorzugt. Dies wird ersichtlich beim Studium aktueller Publikationen zu Neuentwicklungen der Reproduktionsmedizin, in denen die Rechte der werdenden Eltern meist deutlich stärker gewichtet werden [2].

Es mag deshalb wenig erstaunen, wenn der Pädiater Remo Largo in einem Interview mit der Kundenzeitung der Krankenkasse Sanitas die Aussage macht, dass heute viele zukünftige Eltern ihr Kind als Projekt ansähen, für das man sich bewusst entscheide. Damit verbunden seien grosse Erwartungen an den Nachwuchs. Das Kind ist nicht mehr ein Zufallsprodukt, sondern ein vom Zeitpunkt der Zeugung an geplantes Projekt [3]. Ein Kind ist erst dann erwünscht und liebenswert, wenn alle Punkte auf der Checkliste der erwünschten Eigenschaften abgehakt werden können. 
Das Bedürfnis der Eltern und der Gesellschaft nach gesundem Nachwuchs ist gut nachvollziehbar. Im Folgenden soll geklärt werden, ob und wie eugenische Entscheidungen basierend auf immer präziseren Methoden der pränatalen Diagnostik und moderne Vorstellungen von Elternschaft in Einklang gebracht werden können mit Kants Selbstzweckformel.

\section{Motivation hinter eugenischen Entscheidungen}

Die Gesetze der Evolution haben schon immer dafür gesorgt, dass sich Menschen zur Optimierung ihres Nachwuchses den bestmöglichen Partner ausgesucht haben. Dass Eltern Wunschvorstellungen davon haben, welchen Beruf oder welchen Partner ihr Kind wählen soll, und Versuche anstellen, dass ihre Wünsche diesbezüglich erfüllt werden, ist ebenfalls kein Phänomen neuerer Zeit. Es ist auch davon auszugehen, dass sich die meisten Eltern nur das Beste für ihr Kind wünschen.
Wenn also das altruistische Motiv die negative Selektion in zahlreichen Fällen nicht annähernd ausreichend begründet, muss es wohl noch andere ungenannte gewichtige Gründe dafür geben. Artikel 119 des Schweizerischen Strafgesetzbuches sieht vor, dass eine Schwangerschaft straflos abgebrochen werden kann, wenn nach ärztlichem Urteil von der schwangeren Frau die Gefahr einer schwerwiegenden körperlichen Schädigung oder einer schweren seelischen Notlage abgewendet werden kann. Mit einer schwerwiegenden körperlichen Schädigung der Mutter ist nicht zu rechnen, wenn sie ein Kind mit Turner-Syndrom austrägt. Die Schwangere kann also nur eine seelische Notlage geltend machen. Was könnte diese grosse seelische Notlage auslösen? Dass das altruistische Motiv infolge der geringen Beeinträchtigungen von Frauen mit Turner-Syndrom nicht ausreichend sein kann, wurde bereits dargelegt. Die zusätzlichen Gründe können nur egoistisch motiviert sein: Eltern, die sich ein Kind mit besseren Erbanlagen wünschen

\section{Eltern werden im Endeffekt in ihrer Reproduktionsautonomie nicht gestärkt, sondern von der Gesellschaft, die sie für die Geburt eines kranken Kindes verantwortlich macht, unter Druck gesetzt.}

Es fragt sich also, ob sich überhaupt etwas ändern würde, wenn zukünftige Eltern dank nichtinvasiver pränataler Diagnostik zu einem sehr frühen Zeitpunkt der Schwangerschaft der Natur etwas nachhelfen, indem sie Embryonen mit unerwünschten genomischen Aberrationen durch einen medikamentösen Schwangerschaftsabbruch daran hindern, sich weiterzuentwickeln. Dies geschähe zu einem Zeitpunkt, wo viele Embryonen aufgrund von Spontanaborten absterben. Als Begründung könnten die Eltern das altruistische Motiv anführen, dass man dem Kind unnötiges Leiden ersparen will. Die Eltern schrieben sich also die Fähigkeit zu, sich in die Gedankenwelt ihres noch gar nicht geborenen Kindes hineindenken zu können und kommen zum Schluss, dass ein Leben mit dieser Grunderkrankung für ihr Kind nicht lebenswert wäre. Das betroffene Kind erhielte keine Möglichkeit, zu dieser Annahme Stellung zu nehmen und den Eltern das Gegenteil zu beweisen. Mögliche, aber keinesfalls in jedem Fall schwerwiegende Einschränkungen der Lebensqualität bestehen beim Turner-Syndrom aufgrund der erhöhten Missbildungsrate, des Kleinwuchses und der deutlich eingeschränkten Fertilität. Die Lebenserwartung ist aber nicht eingeschränkt, ebenso wenig die intellektuellen Fähigkeiten. Aufgrund dieser Tatsachen besteht guter Grund zur Annahme, dass die meisten Frauen mit Turner-Syndrom ein gutes und erfülltes Leben führen können, wie auch das eingangs zitierte Gedicht belegt. oder dem Druck des Umfelds oder der Gesellschaft nachgeben, die ebenfalls aus egoistischen Motiven auf die negative Selektion eines unperfekten Kindes drängt, das dem Gesundheitssystem vermehrt Kosten verursachen wird.

In den meisten Fällen von selektiver Eugenik wird die Motivlage gemischt sein. Es ist davon auszugehen, dass eigennützige Motive umso mehr überwiegen werden, je früher die pränatale Diagnostik und der darauffolgende eugenische Entscheid erfolgen und je weniger Risiko die verwendete Diagnosemethode für die Mutter und die gesunden und deshalb erwünschten Embryonen beinhaltet. Beide Kriterien sind bei der nichtinvasiven pränatalen Diagnostik erfüllt.

\section{Mögliche Folgen einer liberalen Anwendung eugenischer Massnahmen}

Das ungeborene Kind kann kein Recht auf Existenz einklagen und erleidet keinen Schaden durch seine Nichtexistenz, insbesondere dann nicht, wenn es Träger einer schweren Erkrankung ist, die mit schwerstem Leiden und frühem Tod einhergeht. Insofern scheinen aus Sicht des nicht ausgetragenen Embryos keine negativen Konsequenzen erwachsen zu können. Lediglich zwei der zahlreichen aus ethischer Sicht problematische Konsequenzen einer nicht ausschliesslich altruistischen Motivlage für negative eugenische Entscheide sollen im Folgenden diskutiert werden. 


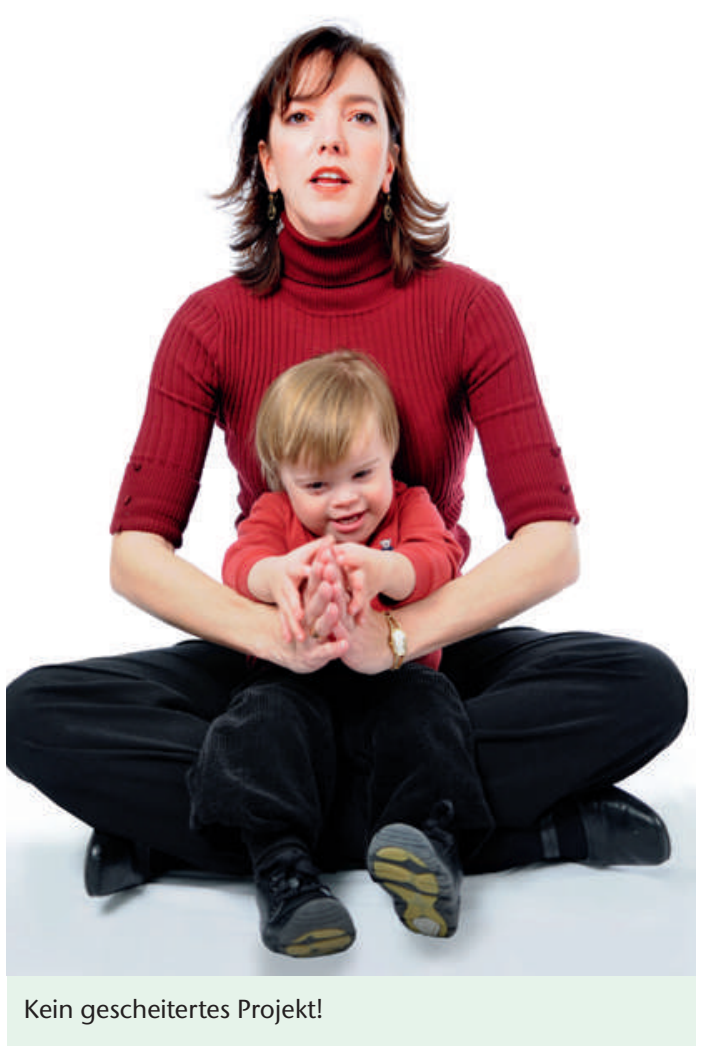

Fliessender Übergang zur positiven Eugenik

Eltern, die eine rein egoistisch motivierte negative Eugenik betreiben, geben den ausgetragenen Kindern die unmissverständliche Botschaft, dass sie ihr Überleben dem Fehlen unerwünschter Erbanlagen verdanken. Die Liebe der Eltern zu ihrem Kind ist von Anfang an eine bedingte Liebe. Die Grenzen zur positiven Eugenik sind fliessend, wenn wie in einigen Fertilitätskliniken in den USA unter dem Begriff Family balancing den behandelten Paaren nicht nur der Kinderwunsch erfüllt wird, sondern auch noch gleich der Traum, das Geschlecht dieses Wunschkindes durch gezielte Auswahl der implantierten Embryonen selber zu bestimmen [4]. In Ländern mit Präferenz für männlichen Nachwuchs wie Indien ist durch die selektive Abtreibung weiblicher Feten in den letzten 20 Jahren ein deutlicher Überschuss männlicher Nachkommen entstanden [5].

\section{Autonomiedefizit bei Nachfolgegenerationen}

Das Bedürfnis nach Kontrolle über den eigenen Nachwuchs wird sich über die Jahre in einer Gesellschaft «gemachter» Individuen perpetuieren und zunehmend verstärken. So weit, dass die eigene Autonomie nur noch am eigenen Nachwuchs ausgelebt werden kann, statt am eigenen Leib. Eine solche Stellvertreterautonomie gefährdet die seit der Antike geltenden Grundlagen moralischen Handelns und das Selbstverständnis der Individuen in einer liberalen Gesellschaft. Sie treibt uns zurück in die selbstverschuldete Unmündigkeit, aus der uns die Aufklärung endgültig befreien wollte. Die erste Generation, die ihrem Nachwuchs die volle Autonomie über das eigene Leben entzieht, trägt damit die Verantwortung für diese unheilvolle Entwicklung.

\section{Eugenische Massnahmen und Kants Selbstzweckformel}

\section{Die Selbstzweckformel}

In der Kritik der Urteilskraft [6] unterscheidet Kant eine äussere Zweckmässigkeit von einer inneren Zweckmässigkeit. Unter äusserer Zweckmässigkeit versteht er alle Dinge der zweckvoll organisierten Natur, die einem anderen als Mittel zum Zweck dienen und somit äusserlich im Verhältnis auf das andere Wesen zweckmässig sind. Einzige Ausnahme bildet der Mensch, der sich dank seiner Vernunft einerseits einen Begriff von Zwecken machen und sich andererseits selber Zwecke setzen kann. Damit ist der Zweck der Existenz eines vernünftigen Wesens in ihm selbst. Es ist nicht nur Zweck, sondern sein Dasein hat den höchsten Zweck in sich selbst, ist Selbstzweck, auf die Formel des kategorischen Imperativs gebracht: «Handle so, dass du die Menschheit sowohl in deiner Person als in der Person eines jeden anderen jederzeit zugleich als Zweck, niemals bloss als Mittel brauchst.» [7]

\section{Verstoss gegen die Selbstzweckformel}

Da wir alle Teil einer zweckmässig organisierten Natur sind, können wir uns gegenseitig bis zu einem gewissen Grad instrumentalisieren, ohne dabei gegen die Selbstzweckformel zu verstossen. Problematisch wird es aber dann, wenn das Gegenüber nicht mehr als vernünftiges, sich selber Zwecke setzendes Individuum mit einem Zweck an sich selbst anerkannt und gewürdigt, sondern als blosses Mittel zum Zweck oder, anders ausgedrückt, als austauschbarer Ermöglichungsgrund einer Handlung missbraucht wird. Die Grenze der Instrumentalisierung wird dann erreicht, wenn dem Gegenüber sein Selbstzweck und damit die Möglichkeit, sich selber Zwecke zu setzen und autonom zu handeln, abgesprochen wird.

\section{Missbrauch des Kindes als Mittel zum Zweck}

Der Verdacht auf Missbrauch des Kindes durch die Eltern als blosses Mittel zum Zweck und damit ein Verstoss gegen das von Kant in der Selbstzweckformel formulierte Instrumentalisierungsverbot trifft umso mehr zu, je geringfügiger die Auswirkungen der kranken Erbanlagen auf das spätere Wohlergehen oder die Vernunftfähigkeit des Kindes sind. Der Verdacht wird zur Gewissheit, wenn es sich um die selektive Elimination von Embryonen oder Feten mit Monosomie X oder weiblichen Geschlechts handelt, bei denen keine Zweifel an der späteren Vernunftfähigkeit des sich entwickelnden Individuums bestehen.

Im Entwurf zum neuen Bundesgesetz über die medizinisch unterstützte Fortpflanzung [8] heisst 
es in Art. 5: «... Die Untersuchung des Erbguts von Embryonen in vitro und deren Auswahl nach Geschlecht oder nach anderen Eigenschaften sind nur zulässig, wenn:

a. die Gefahr, dass sich ein Embryo mit einer Veranlagung für eine schwere Krankheit in die Gebärmutter einnistet, anders nicht abgewendet werden kann; [...]

d. das Paar gegenüber der Ärztin oder dem Arzt schriftlich geltend macht, dass ihm die Gefahr nach Buchstabe a nicht zumutbar ist.»

Der Schweregrad, der ausreichend ist, um einen Embryo zu verwerfen, kann sich also danach richten, ob den Eltern ein Kind mit einer solchen Erkrankung zumutbar ist. Was wird die Gesellschaft über den Wert von Menschen denken, deren Erkrankung für ihre Eltern als unzumutbar gilt? Kinder mit Be- dieses Konzept nicht in Widerspruch gerät zur Forderung, jedem vernünftigen Wesen einen Zweck an sich selbst zuzugestehen und es nicht bloss als Mittel zu betrachten. Dann nämlich, wenn man den Begriff Projekt so auslegt, dass es sich die Eltern zur Aufgabe machen, dem Kind die bestmöglichen Rahmenbedingungen zu bieten, damit es seine Persönlichkeit und seine Fähigkeiten gemäss seinem eigenen Willen und seinen eigenen Lebenszielen zur optimalen Enfaltung bringen kann. Damit bleibt das Recht des Kindes auf einen frei wählbaren selbstbestimmten Lebensweg unter Berücksichtigung der Bedingungen der Realität erhalten. Der Selbstzweckformel Kants wäre Genüge geleistet. Das würde aber auch bedingen, dass Eltern ihr Kind unabhängig von seinen Erbanlagen vorbehaltlos akzeptieren, sofern diese mit einem selbstbestimmten Leben vereinbar sind.

\section{Bedingung wäre, dass Eltern ihr Kind unabhängig von seinen Erbanlagen vorbehaltlos akzeptieren, sofern diese mit einem selbstbestimmten Leben vereinbar sind.}

hinderungen durch Geburtskomplikationen, mit postnatal erworbenen Behinderungen oder Kinder, die wegen unterlassener Pränataldiagnostik mit Behinderung geboren werden, müssen demnach je nach Schweregrad der Behinderung als für ihre Eltern unzumutbar gelten. Die Auffassung vom Menschen als Selbstzweck bleibt aussen vor.

Eltern müssen sich zunehmend vor der Gesellschaft für ein krankes Kind verantworten. Sie werden im Endeffekt dank der Errungenschaften der Reproduktionsmedizin und Pränataldiagnostik in ihrer Reproduktionsautonomie nicht gestärkt, sondern von der Gesellschaft, die sie für die Geburt eines kranken Kindes verantwortlich macht, unter Druck gesetzt. Es entsteht zunehmend die Vorstellung, dass es in jedem Fall möglich ist, ein gesundes Kind hervorzubringen, das einer immer höheren Gesundheitsnorm der Gesellschaft entspricht.

\section{Was wäre zu tun?}

Alternative Interpretation des Projektbegriffs Den Projektbegriff in Zusammenhang mit dem Nachwuchs könnte man auch so definieren, dass

\section{Literatur}

1 Ullrich Turner Syndrom ...na und?! http://turner-syndrom.jimdo.com/\%C3\%BCber-uts/besondere-frauen/

2 Bleichenbacher M, Heitlinger E, Imthurn B. Die Vision von assistierter Fortpflanzungsmedizin in der Schweiz. Schweiz Ärztezeitung. 2010;91(36):13-7.

3 Kundenmagazin Sanitas. www.sanitas.com/dam/ Sanitas/Web/Ueber_Sanitas/Publikationen/Kundenmagazine/Dokumente/DE/Kundenmagazin_3_2011. pdf

4 www.genderselectioncenter.com

5 Jha P, Kesler MA, Kumar R, Ram F, Ram U, Aleksandrowicz L, Bassani DG, Chandra S, Banthia JK. Trends in selective abortions of girls in India: analysis of nationally representative birth histories from 1990 to 2005 and census data from 1991 to 2011. Lancet. 2011;377:1921-8.

6 Kant I. Kritik der Urteilskraft. Art. 82-84. Hamburg: Meiner-Ausgabe edn; 1924.

7 Kant I: Grundlegung zur Metaphysik der Sitten. Hamburg: Meiner-Ausgabe edn; 1965.

8 Laufende Rechtsetzungsarbeiten. www.bag.admin.ch/ themen/medizin/03878/06152/index.html?lang=de

9 Johnson EJ, Goldstein D. Medicine. Do defaults save lives? Science. 2003;302:1338-9. Monosomie 